consultants were really looking for exceptional personal qualities when choosing a candidate, and it would be expected that they would concentrate on less arguable and more specific aspects of the curriculum. In fact, of the most recently appointed senior registrars in psychiatry for the North West Thames Regional Health Authority (Dr Angela Jones, personal communication) those who were appointed in adult psychiatry have had research published and all have completed some research projects. However, only two of the four SRs appointed to mental handicap rotations and only one out of three appointees to child and adolescent psychiatry had published research; this suggests that in certain disciplines the competition is less fierce. There is possibly a conflict for consultants between their original convictions and their practice when selecting candidates. If there is not much difference in the training of the candidates as specified in their curricula vitae, the selectors may scrutinise the research sections of the application forms in search of clues to the aid of short-listing. Consultants may be looking at factors other than research to appoint a candidate, but this will happen after the short-list process has made a selection according to research merits. Regardless of the reason, it is worrying to see that the registrars' response seems to show that they are not really aware of this.

The higher proportion of consultants considering it essential to have managerial skills might be because of the increasing pressure on them to undertake these responsibilities. At present, provisions for management training are restricted to SR level, although it can be speculated that more emphasis will be put on this aspect of registrar training. It is important that trainees discuss their medium-term and long-term career plans with their educational supervisor and clinical tutor early in their training.

Senior registrars should also be encouraged to discuss this matter with more junior colleagues as part of their supervision.

\section{Acknowlegements}

We wish to thank the Merck Research Committee for funding this study, Dr Angela Jones for providing us with valuable information and Dr Shôn Lewis for his comments.

\section{References}

CRISP, A. H. (1990) The case for teaching and research experience and education within basic specialist training (registrar grade) in psychiatry. Psychiatry Bulletin, 14, 163-164.

Junaid, O. \& Staines J. (1990) Career progression in psychiatry: Perceptions and realities. Psychiatric Bulletin. 14, 486-489.

LEWIS, S. (1991) The right stuff? A prospective controlled trial of trainees' research. Psychiatric Bulletin, 15,478-480.

The Royal College of Psychiatrists (1987) Handbook for Inceptors and Trainees in Psychiatry, 86-87.

\title{
The 1994 Senior Registrars Residential Conference
}

The 1994 Senior Registrars Residential Conference will take place at the University of York on 14 to 16 April.

Workshops will include presentation skills, media training, theory and management of aggressive patients, witness skills, research methodology, medical ethics, first day as a consultant, NHS Reforms and writing scientific papers. Further information from Jean Wales at the College. 